REFERENCES:

[1] WHO, COVID-19 clinical management, Living Guidance, 25 Jan 2021 Disclosure of Interests: CODRINA ANCUTA Speakers bureau: Abbvie, Pfizer, Lilly, Novartis, Sandoz, Consultant of: Abbvie, Pfizer, Lilly, Novartis, Sandoz, Cristina Pomirleanu Speakers bureau: Abbvie, Pfizer, Lilly, Novartis, Sandoz, Georgiana Strugariu Speakers bureau: Abbvie, Pfizer, Lilly, Novartis, Sandoz, Luiza Petrariu Speakers bureau: Abbvie, Pfizer, Lilly, Novartis, Sandoz, Eugen Ancuta: None declared, Codruta Bran Speakers bureau: Abbvie, Pfizer, Lilly, Novartis, Sandoz, Rodica Chirieac Speakers bureau: Abbvie, Pfizer, Lilly, Novartis, Sandoz, Claudia Mihailov Speakers bureau: Abbvie, Pfizer, Lilly, Novartis, Sandoz, Consultant of: Abbvie, Pfizer, Lilly, Novartis, Sandoz

DOI: 10.1136/annrheumdis-2021-eular.3467

\section{AB0696 ANTI-PHOSPHOLIPID AUTOANTIBODIES IN COVID-19 PATIENTS}

M. Jordhani', D. Ruci ${ }^{1}$, V. Ruci'. ${ }^{1}$ UHC Mother Teresa, Tirana, Albania, Internal Medicine, Tirana, Albania; ${ }^{2}$ University Trauma Hospital Tirana, Orthopaedics, Tirana, Albania

Background: Because of the inflammation boosting cytokines, Coronavirus disease 2019 (COVID-19) has demonstrated thrombotic consequences that have increased its morbidity and mortality. There is evidence that mechanisms that contribute in thrombosis in COVID-19 patients are similar to those in anti-phospholipid syndrome (aPS). In fact, there is a possibility that anti-phospholipid autoantibodies (aPLs) might impulse thrombosis in patients with COVID-19, as literature suggests ${ }^{2}$.

Objectives: The aim of our study was to evaluate the anti-phospholipid autoantibody titre in patients with COVID-19 during and after the infection.

Methods: This is an observational study which included 71 patients with a recent COVID-19 up to 4 weeks after. Every patient was completed with aPL titre about IgG and IgM anti-cardiolipine (ACA) and lupus anticoagulant (LAC) autoantibodies. According to titre results, the patients were divided into groups in order to better show the immunologic results.

Results: After gathering and analysing the data, it was estimated that 21 patients (29.6\%) were positive for at least one type of aPL antibody: 12 patients were found positive for lupus anticoagulant autoantibodies (57.1\%), 6 patients were double positive for LAC and ACA $(28.6 \%)$, and 3 patients were positive for anti-cardiolipin antibodies (14.3\%). Seven patients were IgM positive for any aPL (33.3\%), 6 patients were found to have positive $\lg$ M and $\operatorname{lgG}(28.6 \%)$ and 8 patients had only IgG antibodies (38.1\%).

Conclusion: From this study it was observed that a significant proportion of patients with recent COVID-19 infection had positive anti-phospholipid antibodies, compared to the general population prevalence. This suggests that the impact of aPLs in COVID-19 might be of great importance. It should be carefully evaluated in order to better understand the mechanisms of thrombotic complications.

REFERENCES:

[1] Wise Jacqui. Covid-19 and thrombosis: what do we know about the risks and treatment? BMJ 2020; 369:m2058

[2] Zuo, Yu, et al. "Prothrombotic autoantibodies in serum from patients hospitalized with COVID-19." Science translational medicine 12.570 (2020).

Disclosure of Interests: None declared

DOI: 10.1136/annrheumdis-2021-eular.3521

\section{AB0697 ANTI-SARS-COV-2 ANTIBODIES AND AUTOANTIBODIES IN COVID-19 PATIENTS SURVIVED AFTER ICU ADMISSION, 6 MONTHS LATER}

K. Bitzogli ${ }^{1}$, E. Magira ${ }^{2}$, L. Chatzis' ${ }^{1}$, E. Jahaj ${ }^{2}$, H. Alexopoulos ${ }^{1}$, M. Dalakas ${ }^{1}$, A. Kotanidou', A. Tzioufas ${ }^{1}$, P. Vlachoyiannopoulos ${ }^{1} .^{1}$ School of Medicine, National and Kapodistrian University of Athens, Pathophysiology, Athens, Greece; ${ }^{2}$ School of Medicine, National and Kapodistrian University of Athens, 1st Department of Intensive Care Medicine, Athens, Greece

Background: $\mathrm{We}^{1}$ and others ${ }^{2}$ have previously shown that ICU admitted patients with COVID-19 developed high titers of anti-SARS-CoV-2 antibodies, but also autoantibodies, some of which are pathogenic. We re-evaluated 8 patients of those survived after admission to the ICU of Evangelismos Hospital of Athens $-1^{\text {st }}$ Department of Internal Medicine, Medical School, NKUA ${ }^{3} 6$ months later. We did not know whether these autoantibodies still exist, are associated with COVID19 or with ARDS as described after septic shock ${ }^{4}$.

Objectives: To investigate the presence and titers of anti-SARS-CoV-2 antibodies and autoantibodies in patients survived after COVID-19 ICU stay, in the ICU and 6 months later.

Methods: Case series to evaluate titers of anti-SARS-CoV-2 antibodies, specificities of autoantibodies as well as clinical features in ICU admitted COVID-19 patients, initially and 6 months after their discharge. Evaluation of current clinical status included evaluation of lung, heart, kidney, central and peripheral nervous system and mental status using standardized methods. Methods for detection of anti-SARS-CoV-2 antibodies and autoantibodies were described in our previous report ${ }^{1}$

Results: We had initially evaluated ${ }^{1} 29$ ICU admitted COVID-19 patients' files and sera, of which 4 had been already died during serum evaluation. Six more patients died thereafter. Out of 19 having been discharged, 8 were willing to be re-evaluated. On second evaluation 6 months later, serum anti-SARS-CoV-2 antibodies were highly positive, although at lower titers compared to the titers at disease onset (median [range]) 8.705 (range: 7.95-9.56) vs 6.640 (range: 6.29 6.76), $p=0.0002$, Mann-Whitney test. Initially 3 out of 8 patients expressed antinuclear antibodies (ANA) at titers $1 / 160,1 / 320$ and $1 / 320$ with a fine speckled pattern with the second patient also expressing at a titer of $1 / 160$, antimitochondrial (AMA) antibodies. Six months later the same patients and not anyone else expressed ANA of the same pattern at titers $1 / 640,1 / 160$ and $1 / 160$ respectively. Two patients with $1 / 20 \mathrm{p}$-ANCA and $1 / 640 \mathrm{c}$-ANCA initially, lost their respective autoantibodies after 6 months. One patient initially negative for IgM anti-ß2GP became positive at low titer and an initially positive became negative. One patient initially positive for anti-Ro60 antibody continued to be positive 6 months later. One patient initially negative developed anti-Tg antibodies and 3 patients initially positive for anti-TPO antibodies remained positive 6 months later.

Conclusion: Patients with COVID-19 survived after ICU admission still retain high titers of anti-SARS-CoV-2 antibodies but significantly lower that at disease onset, but they tend to lose autoantibodies with pathogenic potential.

\section{REFERENCES:}

[1] Vlachoyiannopoulos P et al, Ann Rheum Dis 2020,

[2] 2. Wang $E Y$ et al, medRxiv preprint doi: https://doi. org/10.1101/2020.12.10.20247205

[3] National and Kapodistrian University of Athens, Athens, Greece

[4] Burbelo et al. Journal of Translational Medicine 2010

Disclosure of Interests: None declared

DOI: 10.1136/annrheumdis-2021-eular.3554

\section{AB0698 CORONAVIRUS 19 DISEASE VACCINE: PERCEPTIONS AND INTENTIONS OF TUNISIAN PATIENTS WITH RHEUMATOID ARTHRITIS}

K. Ben Abdelghani ${ }^{1}$, H. Boussaaa ${ }^{1}$, S. Miladi ${ }^{1}$, M. Sellami ${ }^{1}$, L. Souabni ${ }^{1}$, K. Ouenniche ${ }^{1}$, S. Kassab ${ }^{1}$, S. Chekili', A. Fazaa ${ }^{1}$, A. Laatar ${ }^{1} .{ }^{1}$ Mongi Slim University Hospital, Rheumatology, La Marsa, Tunisia

Background: Coronavirus disease 2019 (covid-19) has now spread to millions of persons worldwide to become a global pandemic. Covid-19 is asymptomatic for some individuals and for others it can cause symptoms ranging from flu-like to acute respiratory distress syndrome, pneumonia and death. Several vaccine candidates are now available, and patients with chronic inflammatory disease such as rheumatoid arthritis (RA) are encouraged to get vaccinated.

Objectives: The aim of this study was to determine perceptions of RA patients about the covid-19 vaccine.

Methods: We conducted a cross-sectional study including Tunisian patients with RA (ACR/EULAR 2010). Demographic and disease parameters were collected: age, gender, educational status, disease duration, erythrocyte sedimentation rate (ESR), disease activity score (DAS28), and treatments being used. All patients responded to a questionnaire on their perceptions and concerns about the covid-19 vaccine, and whether they intended to get vaccinated or not. A $p$ value inferior to 0.05 was considered significant.

Results: We included 54 patients ( 45 women and nine men) with a mean age of $55 \pm 11$ years old [23-69]. Thirty-one percent of patients were illiterate. The mean disease duration was $9.9 \pm 5.9$ years $[0-20]$. The mean DAS28 ESR was $4.68 \pm 1.35$ [1.50-7.16].

NSAIDs were used in $13 \%$ of patients, corticosteroids in $63 \%$ of patients at a mean daily dose of $8 \mathrm{mg}$ [2.5-20] of prednisone equivalent, methotrexate in $63 \%$ of patients, sulphasalazine in $13 \%$ of patients, leflunomide in $3.7 \%$, and biologics in $22.2 \%$ of patients.

None of these patients had contracted the covid-19. Seventeen percent of patients had been in close contact with someone positive for the disease. All the patients reported that they respected the preventive measures. Fifteen percent of patients had stopped their treatment because they were afraid of the covid19: Methotrexate $(n=4)$, sulphasalazine $(n=1)$, tocilizumab $(n=2)$, and rituximab $(n=1)$.

More than half of patients $(67.7 \%)$ reported that they didn't want to get vaccinated against covid-19. The reasons given by these patients were: presumed adverse events $(100 \%)$, religious beliefs $(86.7 \%)$. presumed inefficiency $(83.3 \%)$, no recommendation from their doctor $(80 \%)$, fear that the vaccine would interact with their treatment $(76.7 \%)$, lack of trust in the pharmaceutical laboratories $(76.7 \%)$, fear from vaccines in general $(53.3 \%)$, fear that the vaccine would make RA worse $(50 \%)$, and presumed overprice of the vaccine $(30 \%)$. 
A significant association was noted between educational status and, religious beliefs $(p=0.001)$, lack of trust in the pharmaceutical laboratories $(p=0.007)$, fear that the vaccine would make RA worse $(p=0.008)$ or interact with other treatments $(p=0.022)$, and presumed overprice of vaccine $(p<0.001)$.

Conclusion: Most of RA patients expressed their unwillingness to get vaccinated against covid-19. Doubts about the vaccine's efficiency and security and religious beliefs were the main reasons for this choice.

Disclosure of Interests: None declared

DOI: 10.1136/annrheumdis-2021-eular.3667

\section{AB0699 RETROSPECTIVE ANALYSIS OF 52 SARS-COV2 POSITIVE PATIENTS WITH INFLAMMATORY RHEUMATIC DISEASE: A SINGLE CENTER EXPERIENCE}

Y. Karabulut ${ }^{1}{ }^{1}$ Private Yıldırım Doruk Hospital, Division of Rheumatology, Bursa, Turkey

Background: It is a generally accepted opinion that autoimmune and inflammatory rheumatic diseases or drugs used in the treatment of these conditions increase the risk of infection. During the pandemic period, the follow-up and treatment of patients who were diagnosed with rheumatic disease and used corticosteroid, immunosuppressive, biological or synthetic DMARDs and disease management during sars-cov2 infection still remain a problem.

Objectives: In this study, it was aimed to share the demographic data of 52 patients with inflammatory rheumatic disease diagnosed with SARS-COV2 who were followed up and treated in the Rheumatology Department of Private Doruk Hospital during the SARS-COV2 pandemic. Additionally, it is aimed to examine the primary rheumatological diseases of the patients, their biological and conventional DMARD treatments, their comorbidities and the course of SARS-COV2.

Methods: Fifty-two patients who were diagnosed with SARS-COV2 by PCR method while being followed up and treated in the rheumatology center between May 2020 and November 2020 and get COVID treatment in the same center were included in the study. All patients diagnosed with SARS-COV2 and required hospitalization were hospitalized in the same center and followed up and treated. The files and electronic records of the patients were retrospectively recorded by the rheumatologist who followed the patients.

Results: In this retrospective study conducted from a single center, 52 patients whose diagnosis of SARS-COV2 was confirmed by PCR were included. Distribution of primary rheumatic diseases of the patients; 19 rheumatoid arthritis (RA), 14 Ankylosing spondylitis (AS), 4 Psoriatic Arthritis (PsA), 4 Systemic lupus erythematosus (SLE), 5 Behcet's Disease (BD), 4 Familial Mediterranean Fever (FMF), 2 Sjögren's syndrome. $76.8 \%$ of the patients were female, $22.2 \%$ male, their mean age was $47 \pm 18$. Biological drug use rate of 37 patients in RA, AS and PsA groups was $83.7 \%$ (monotherapy or combination 31/37).

Moreover, $16.2 \%(6 / 37)$ of the patients were using synthetic DMARD combination (MTX+SLZ $+\mathrm{HCQ}$ ) and $40.5 \%(15 / 37)$ of the patients were using a combination of biological and synthetic DMARDs. While $73 \%(38 / 52)$ of 52 patients had a mild course, 27\% (14/52) had severe SARS-COV2 requiring hospitalization. 14 patients who had severe SARS-COV2 infection and required hospitalization, 10 were followed up with the diagnosis of RA, 2 with AS and 2 with SLE. Hospitalization of patients using monotherapy biological drugs (TNF inhibitor, Tocilizumab, IL 17-A) due to severe SARS-COV2 was found to be lower than the group using combined synthetic DMARDs with steroids $(\mathrm{MTX}+\mathrm{SSZ}+\mathrm{HCQ})(\mathrm{p}<0.05)$. The corticosteroid dose of the RA patients was in the range of $5-10 \mathrm{mg} / \mathrm{day}$. The rate of having severe SARS-COV2 was found to be higher in the combination group using biological or synthetic DMARD and low dose corticosteroids compared to group using monotherapy biologicals $(p<0.05)$. The rate of having severe SARSCOV2 was found to be significantly higher in the group using 10/mg or more at the time of diagnosis $(p<0.05)$. Two patients with SLE multiple organ involvement had severe SARS-COV2 while using rituximab, and hospitalization was required. In terms of comorbidities, hypertension was the most common comorbidity with $64.2 \%(9 / 14)$ in the group with severe SARS-COV2, followed by obesity with $21.4 \%(3 / 14)$

Conclusion: In patients with inflammatory rheumatic disease, SARS-COV-2 infection and the drugs used for the treatment of primary disease are still considered to be a difficult situation in terms of prognosis. In our study with limited cases, data suggesting that there is no increased risk of SARS-COV2 requiring hospitalization in patients using TNF inhibitors, tocilizumab and IL17-A blockers. It was thought that there might be a drug-induced increased risk due to the severe SARS-COV2 infection that developed in our two patients who used rituximab, but the disease-related risk increase was not ignored because the patients were SLE patients with active multi-organ involvement.

Disclosure of Interests: None declared

DOI: 10.1136/annrheumdis-2021-eular.3672

\section{$\mathrm{AB} 0700$ TOCILIZUMAB DID NOT REDUCE MORTALITY IN SEVERE COVID-19 PATIENTS BUT CAUSED THROMBOCYTOSIS}

Z. Öztürk ${ }^{1}$, S. M. Türk ${ }^{1}$, D. Karataș ${ }^{1}$, Ü. Erkorkmaz ${ }^{2}$, K. Özmen Süner ${ }^{3}$, H. Dheir ${ }^{4}$, E. Güçlü ${ }^{5}$, E. Gönüllü ${ }^{1}$, O. Karabay ${ }^{5}$. ${ }^{1}$ Sakarya University Training and Research Hospital, Rheumatolojı, Adapazarı, Turkey; ${ }^{2}$ Sakarya University Training and Research Hospital, Biostatistics, Adapazarı, Turkey; ${ }^{3}$ Sakarya University Training and Research Hospital, Anesthesia, Adapazarı, Turkey; ${ }^{4}$ Sakarya University Training and Research Hospital, Nephrology, Adapazarl, Turkey; ${ }^{5}$ Sakarya University Training and Research Hospital, Infectious Diseases and Microbiology, Adapazarı, Turkey

Background: TCZ is a monoclonal antibody against Interleukin-6 receptor (IL$6 \mathrm{R})$ which is used for relieving inflammation and reducing mortality in COVID 19 patients. Safety and efficacy of Tocilizumab (TCZ) in Covid-19 pneumonia is uncertain yet. In this study, we aimed to determine clinical outcomes in patients treated with $\mathrm{TCZ}$

Objectives: In this study we aimed to share our retrospective results which we had obtained from patients with COVID-19 diagnosis received TCZ.

Methods: We performed a retrospective case control study between May and August 2020 in Turkey. We compared outcomes in patients who received TCZ with those who did not. Death in hospital and intensive care unit (ICU) requirements were evaluated as endpoints. Demographic data, comorbidities, additional treatment, treatment side effects, laboratory and clinical results were retrospectively assessed. There are no significant differences between groups according to age, gender and Charlson Comorbidity Index (CCl).

Results: 12 (27.3\%) patients died in standard group and eight (18.6\%) patients died in TCZ group ( $p=0.150)$.Days of staying in the hospital were eight days in standard treatment group and 12 days in TCZ group $(p=0.03) .10$ of 43 patients in TCZ group were admitted to ICU. MV support was needed in 8 of these patients. 18 of 44 patients $(40.9 \%)$ within the standard group were admitted to ICU and 12 patients $(27.3 \%)$ were intubated $(p=0.125, p=0.480)$. Significant IL-6 decrease was not observed post treatment in TCZ group according to pretreatment period $(p=0.60)$. Significant decreases were examined in CRP and ferritin values through TCZ treatment. However, D-dimer and thrombocyte values increased.

Conclusion: TCZ may not be an effective treatment for reducing ICU requirement, to prevent intubation or death, for shortening period for staying in hospital. The patients should be followed up closely for possible thrombosis because of increased D-dimer and thrombocytes with TCZ treatment.

\section{REFERENCES:}

[1] Sharma A, Tiwari S, Deb MK, Marty JL. Severe acute respiratory syndrome coronavirus-2 (SARS-Cov-2): A global pandemic and treatment strategies. IntJ Antimicrob Agents. 2020 Aug; 56(2):106054.

[2] Singhal T. A rewiev of coronavirus Disease-2019(COVID-19). Indian J Pediatr. $2020 \mathrm{Apr} ; 87(4): 281-286$.

[3] Mehta P, McAuley DF, Brown M, Sanchez E, Tattersall R.S, Manson J.J. COVID-19: consider cytokine storm syndromes and immunosuppression. Lancet. 2020;395(10229):1033-

[4] Teijaro J.R. Cytokine storms in infectious diseases. Seminlmmunopathol. 2017;39:501-503.

[5] Zhang Y, Li J, Zhan Y, Wu L, Yu X, Zhang W et al. Analysis of Serum Cytokines in Patients with Severe Acute Respiratory Syndrome. Infect Immun 2004 Aug;72(8):4410-4415.

[6] Zhang C, Wu Z, Li JW, Zhao H, Wang GQ. Cytokine release syndrome in severe COVID-19: interleukin-6 receptor antagonist tocilizumab may be the key to reduce mortality. Int J Antimicrob Agents. 2020 May; 55(5):105954.

[7] Xu Z, Shi L, Wang Y, Zhang J, Huang L, Zhang $C$ et al. Pathological findings of COVID-19 associated with acute respiratory distress syndrome. Lancet Respir Med. 2020;8(4):420-2

[8] Fu B, Xu X, Wei H. Why tocilizumab could be an effective treatment for severe COVID-19? J TransI Med 18,164 (2020)

[9] Guaraldi G, Meschiari M, Cozzi-Lepri A, Milic J, Tonelli R, Menozzi M et al. Tocilizumab in patients with severe COVID-19: a retrospective cohort study. Lancet Rheumatol. 2020 Aug;2(8):e474-e484.

[10] Gupta S, Wang W, Hayek S.S, Chan L, MathewsK.S, Melamed M.L et al. Association Between Early Treatment With Tocilizumab and Mortality Among Critically III Patients With COVID-19. JAMA Intern Med. 2021 Jan1;181(1):41-51.

[11] Campochiaro C, Della-Torre E, Cavalli G, De Luca G, Ripa M, Boffini N et al Efficacy and safety of tocilizumab in severe COVID- 19 patients: a single-centre retrospective cohort study. Eur J Intern Med. 2020 Jun;76:43-49.

Disclosure of Interests: None declared

DOI: 10.1136/annrheumdis-2021-eular.3697 